\title{
Determination of Generator Groupings For an Islanding Scheme in the Manitoba Hydro System Using the Method of Normal Forms
}

\author{
V. Vittal W. Kliemann \\ Iowa State University \\ D. G. Chapman A. D. Silk
Manitoba Hydro
}

Abstract: This paper deals with the application of the method of normal forms [1-5] in the analysis of a specific aspect of system dynamic behavior in the Manitoba Hydro system. Following a major loss of transmission capacity on the Manitoba Hydro HVDC system (Nelson River system), and the subsequent operation of protection systems, there is a major deficit of generation in the remaining system, comprising Manitoba and Saskatchewan. The method of normal forms is applied to determine the natural groupings which are formed by the machines in Manitoba Hydro due to nonlinear interaction. This grouping then provides a basis for developing a systematic procedure to island the remaining system.

\section{Introduction}

Recent work [1-5] done by the authors and the group at Iowa State University has dealt with the development of the method of normal forms to analyze the dynamic performance of power systems. The method of normal forms is a well established technique in dynarnical system theory. The unique aspect of this technique is that under certain non resonance conditions, it provides a nonlinear transformation which transforms the original nonlinear system into an equivalent linear system. In addition, the transformation also provides an approximate closed form solution of the system state variables. This solution includes the higher order nonlinear terms, and also provides an approach to identify nonlinear interaction of the fundamental modes of oscillation.

This paper deals with the application of the analytical techniques developed earlier to analyze a specific aspect of system cynamic behavior in the Manitoba Hydro system. In this system, following a major loss of the HVDC system, there is a severe deficit of generation. Protection schemes trip ties between Manitoba Hydro and the surrounding systems. In the

PE-370-PWRS-0-12-1997 A paper recommended and approved by the IEEE Power System Dynamic Performance Committee of the IEEE Power Engineering Societty for publication in the IEEE Transactions on Power Systems. Manusiript submitted August 1, 1997; made available for printing December 12, 1997.

\author{
$\mathrm{Y}-\mathrm{X} \mathrm{Ni}$ \\ University of Hong Kong \\ D. J. Sobajic \\ EPRI
}

remaining portion of the system which consists of Manitoba and Saskatchewan, the serious deficit in generation causes severe underfrequency conditions. As a mitigation step, consideration is being given to the development of a scheme which would split the Manitoba Hydro system into independent islands.

The objective of this paper is to apply the method of normal forms to the remaining Manitoba and Saskatchewan systems, following the total loss of the HVDC system, and investigate whether the various indices and techniques developed will predict a natural grouping which would be formed by the machines. This grouping, which would account for the nonlinear behavior of the system and the nonlinear interaction between modes of oscillation, would then provide a basis for developing a systematic procedure to create islands in the remaining system.

The power system data obtained from Manitoba Hydro, consisted of over 300 generators and 5000 buses. This system represented Manitoba Hydro and Saskatchewan together with the interconnections to the U.S. and Ontario Hydro. The normal form analysis was conducted on only the Manitoba Hydro Saskatchewan systems following the loss of the HVDC system in Manitoba Hydro. The interconnections with the U.S. and Ontario Hydro were represented as shunt impedances at the boundary buses. These shunt impedances accounted for the adjusted flow in the tie line with Ontario Hydro and the U.S. following the loss of the HVDC system.

The Manitoba and Saskatchewan systems are represented by 37 generators, 14 in Manitoba and 23 in Saskatchewan. Of these 9 generators are represented by detailed two-axis models and have static exciters. The remaining generators are represented using the classical model.

Section II briefly describes the mathematical model, and provides an overview of the method of normal forms. Section III presents the procedure applied to the Manitoba Hydro system. Sample results are presented in Section IV. Conclusions are provided in Section V. 


\section{Mathematical Model}

The modeling features in this paper include the following:

Synchronous Machines:

The generators are modeled using either the two axis model [6, Chapter 4] or the classical model [6, Chapter 2]. The use of these models in the method of normal forms is detailed in [1-5].

Excitation Control:

A static exciter model is used to represent the excitation system. The representation of this model in the method of normal forms is provided in detail in $[1,4,5]$.

Load Model:

All load are modeled as constant impedances.

With all the components modeled, the system equations take the form [1-5]

$\dot{x}=f(x)$

Moving the origin to the post disturbance stable equilibrium point (SEP2), taking generator 1 as rotor angle reference, the Taylor's series expansion up to the second order will be of the form

$$
\dot{\boldsymbol{x}}=\boldsymbol{A} \boldsymbol{x}+\boldsymbol{X}_{2}(\boldsymbol{x})
$$

where $\boldsymbol{A}$ is the system Jacobian matrix, $\boldsymbol{X}_{2}(\boldsymbol{x})$ is a vector with each element as a quadratic function of $x$, i.e., $\boldsymbol{X}_{2 i}(\boldsymbol{x})=\frac{1}{2} \boldsymbol{x}^{T} \boldsymbol{H}^{i} \boldsymbol{x}$, where $\boldsymbol{H}^{i}$ is the Hessian matrix given by $\boldsymbol{H}^{i}=\left[\partial^{2} f_{i} / \partial x_{j} \partial x_{k}\right]_{S E P 2}$.

The normal forms technique [1-5] using the second order approximation can now be applied to equation (2).

\section{Normal Forms Technique}

The normal forms of vector fields is used in the study of system behavior after the de system has been shut down. The analysis is conducted using a Taylor's series expansion of the system around a fictitious equilibrium point (Section III) obtained after the loss of the dc system by appropriately adjusting the generation. The procedure to apply the method of Normal Forms to the system given by (2) is given in [1, Section 3.3]. The above references also describe the steps in determining the closed form solutions of the system state variables.

\section{Indices of System Dynamic Behavior}

The following indices, developed and described in earlier work, are used to analyze the system dynamic behavior using the method of normal forms:

1. Linear Participation Factors [7].

2. Second order Participation Factors [3-5]. These are obtained using the second order information from the normal forms. The second order participation factor has the form

$p 2_{k p q}=u 2_{k p q}\left(V_{p k}+v 2_{p k k}\right)\left(V_{q k}+v 2_{q k k}\right)$

where, $V_{i j}$ is the left eigenvector, and $u 2_{i j k}$ and $v 2_{i j k}$ are the terms involving right and left eigenvector elements together with the second order normal form coefficients.

3. Nonlinear Interaction Index Index $1[2,5]$ and the associated procedure to determine the critical modes.

4. Nonlinear Dominance Index Index2 $[2,5]$

\section{Procedure to Determine Generator Grouping}

The disturbance considered is a loss of the de system. This disturbance results in a large portion of generation becoming isolated from the rest of the system. As a result, there is a dearth of generation in the system, and the remaining generators in the system decelerate. In addition, the flows into Manitoba on the tic lines increase. The tie line protection systems, sensing this large change, quickly disconnect the tie lines to protect voltages in the U.S. This results in a further deficit of generation and due to the severe under frequency situation, the Manitoba and Saskatchewan areas are broken up into islands.

The study consists of analyzing the Manitoba and Saskatchewan area immediately following the loss of the dc system, using the method of normal forms. The objective of the study is to use the indices and analytical tools dealing with the normal forms method to analyze the system and determine the natural groups formed by the Manitoba and Saskatchewan areas following the loss of the $\mathrm{dc}$ system. This determination includes the effect of the nonlinear interactions and takes into account the structural characteristics of the system.

The method of normal forms is applied to the Manitoba and Saskatchewan areas after the loss of the dc system. This is done by determining a suitable post disturbance equilibrium point after the loss of the dc system and before the tie lines with the neighboring systems are lost. The Manitoba area is 
shown in Figure 1. The procedure to determine the post disturbance ecuilibrium point is described below.

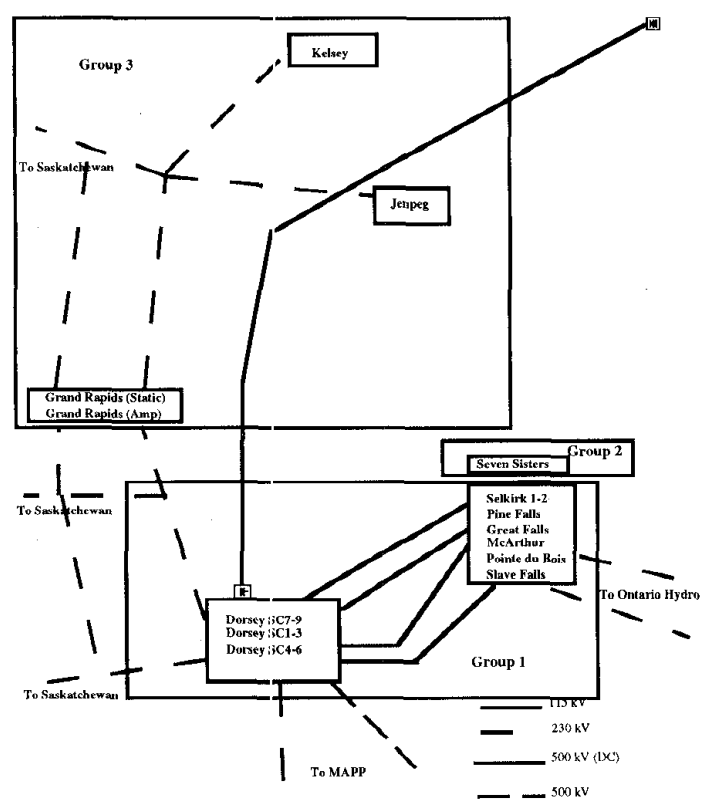

Fig. 1 Study Area in Manitoba

Table 1 shows the bus numbers and names of the generators retained in Manitoba and Saskatchewan The machines at Dorsey are synchronous condensors.

Procedure:

Step 1: Obtain the pre-disturbance stable equilibrium point of the original system (SEPO).

The original system data obtained from Manitoba Hydro consisted of over 300 generators and 5000 buses. This power flow solution is denoted as SEPO.

Step 2: Obtain the pre-disturbance stable equilibrium point of the reduced system (SEP1).

We reduce the original system to a system including only the Manitoba and Saskatchewan areas, with tie line injection powers and $\mathrm{dc}$ injection powers represented as shunt $G_{s}+j B_{s}$ 's at the corresponding buses, and reran the power flow. This is the predisturbance stable equilibrium point (SEP1) of the reduced system.

Step 3: Obtain the post disturbance stable equilibrium point $\sigma^{*}$ the reduced system (SEP2). Using the original system and its pre-disturbance stable equilibrium point we run a time simulation using ETMSP [8]. The HVDC system is disconnected at $\mathrm{t}=\mathrm{C}^{+}$, and the system dynamics are simulated until $0.1 \mathrm{sec}$. This disturbance results in a
Table 1 Generator Bus Names

\begin{tabular}{|c|c|c|c|}
\hline \multicolumn{2}{|c|}{ Generators in Manitoba } & \multicolumn{2}{|c|}{ Generators in Saskatchewan } \\
\hline $\begin{array}{c}\text { Generator } \\
\text { Bus \# }\end{array}$ & $\begin{array}{c}\text { Generator Bus } \\
\text { Name }\end{array}$ & $\begin{array}{c}\text { Generator } \\
\text { Bus \# }\end{array}$ & $\begin{array}{c}\text { Generator Bus } \\
\text { Name } \\
\end{array}$ \\
\hline 3581 & Dorsey SC 7-9 & 3001 & Boundary Dam 1 \\
\hline 3585 & Dorsey SC 1-3 & 3002 & Boundary Dam 2 \\
\hline 3586 & Dorsey SC 4-6 & 3003 & Boundary Dam 3 \\
\hline 3587 & Selkirk $1-2$ & 3004 & Boundary Dam 4 \\
\hline 3588 & Jenpeg & 3005 & Boundary Dam 5 \\
\hline 3590 & Kelsey & 3006 & Boundary Dam 6 \\
\hline 3591 & $\begin{array}{c}\text { Grand Rapids } \\
\text { (Static) }\end{array}$ & 3010 & Poplar River 1 \\
\hline 3592 & $\begin{array}{l}\text { Grand Rapids } \\
\text { (Amplidyne) }\end{array}$ & 3011 & Poplar River 2 \\
\hline 3595 & Pine Falls & 3021 & Shand \\
\hline 3596 & Great Falls & 3032 & E.B. Campbell 1 \\
\hline 3597 & McArthur & 3033 & E.B. Campbell 2 \\
\hline 3598 & Seven Sisters & 3040 & $\begin{array}{c}\text { Queen Elizabeth } \\
1 \\
\end{array}$ \\
\hline 3599 & Pointe du Bois & 3041 & Queen Elizabeth2 \\
\hline 3600 & Slave Falls & 3042 & Queen Elizabeth3 \\
\hline & & 3050 & Nipawin 1 \\
\hline & & 3051 & Nipawin 2 \\
\hline & & 3060 & Island Falls 1 \\
\hline & & 3061 & Island Falls 2 \\
\hline & & 3070 & Coteau Creek 1 \\
\hline & & 3071 & Coteau Creek 2 \\
\hline & & 3072 & Coteau Creek 3 \\
\hline & & 3082 & Meadow Lake \\
\hline
\end{tabular}

severe deficit of generation, since the loss of the dc system isolates a large amount of generation. As a result, the remaining generators decelerate. The decelerating power of the generators in Manitoba and Saskatchewan, $P_{G d e c, i}$, and the tie line injection powers, $P_{t i e, k}$, into the Manitoba and Saskatchewan area at 0.1 second are recorded.

We assume that $50 \%$ of the total decelerating power of the generators in Manitoba Hydro/Saskatchewan area is picked up by the generators in that area according to the machine inertias, referred to a 100 MVA base, i.e.,

$$
\Delta P_{G i}=0.5 \times \sum P_{G d e c, i} \times \frac{H_{i}}{H_{T}}\left(H_{T}=\Sigma H_{i}\right)
$$

$\Delta P_{G i}$ is the amount of generation change at the remaining generators. This number is fixed at $50 \%$, because the deficit of generation is so large that the shortage is greater than the remaining capacity in Manitoba and Saskatchewan. The other $50 \%$ is picked up by the external system. This is represented by modifying the tie line injection power according to the following equation

$$
\Delta P_{t i e, k}=0.50 \times \sum P_{G d e c, i} \times \frac{P_{t i e, k \mid t=0.1 \mathrm{sec}}}{\sum_{j} P_{t i e, j \mid t=0.1 \mathrm{sec}}}
$$


$\sum P_{G d e c, i}$ and $\Delta P_{G i}, \Delta P_{t i e, k}$ are calculated. The resultant $P_{t i e, i}=P_{t i e, i l t=0^{+}}+\Delta P_{i i, i}$ is represented as a shunt $G_{j i}+j B_{j i}$ at the boundary bus. Since in this case the loss in generation is too large for the remaining capacity in Manitoba and Saskatchewan areas, we apportion only $50 \%$ of the shortfall to be picked up by this area. The remaining generation comes over the ties with the surrounding areas. The generator output $P_{G i}$ for the reduced system is modified as shown above. The injection $\left(P_{d}+j Q_{d}\right)$ of the dc system is disconnected and the power flow is rerun to obtain the post-disturbance stable equilibrium point SEP2. This equilibrium point represents the steady state conditions which would exist if the generation was rescheduled in the period between the loss of the dc system and the opening of the tie with the neighboring system.

Step 4: Perform the normal forms calculations and calculate the indices of system dynamic behavior and perform analysis to determine the machine groupings.

\section{Numerical Results}

The procedure developed in Section III was applied to the Manitoba and Saskatchewan systems. Several operating conditions were analyzed. The results for one specific case, based on a typical Winter Load level, with the system intact, are presented below.

The normal forms analysis generates a description of the response of the system which is made up of a linear components and non-linear components in the form

$$
y_{j}(t)=z_{j 0} e^{\lambda_{j} t}+\sum_{k=1 l=k}^{m} \sum_{k}^{m} h 2_{k l}^{j} z_{k 0} z_{10} e^{\left(\lambda_{k}+\lambda_{l}\right) t}
$$

Where the variables $y_{i}$, are the Jordan form variables, $z_{j}$ are the normal form variables, and $h 2_{k \ell}^{j}$ is the normal form coefficient. In conjunction with this solution, we define two indices $[2,5]$.

$$
\text { Index } 1=\left|\left(y_{j 0}-z_{j 0}\right)+\max _{k, i} h 2_{k l}^{j} z_{k 0} z_{l 0}\right|
$$

Where the quantities $y_{j 0}$ and $z_{j 0}$ are the values of the Jordan form and Normal Form variables at time $\mathrm{t}=\mathrm{O}^{+}$. Index 1 provides a measure of the effect of the nonlinear terms in the solution by comparing the linear solution to the second order solution.

$$
\text { Index } 2=\frac{\max _{k, l}\left|h 2_{k l}^{j} z_{k 0} z_{l 0}\right|}{\left|z_{j 0}\right|}
$$

Index 2 determines whether the nonlinear effects arise from the second order terms indicating a strong modal interaction, or whether the second order terms affect the initial solution in the sariables indicating a dominant fundamental mode.

Table 2 shows the inertial modes picked up according to the sorting of the nonlinear interaction index Index 1 . Hence, Index 1 is used to determine the most critical modes with respect to nonlinear interaction among all the modes of the system. Here, the modes are presented in numerical order. The table shows the angular frequency $\omega_{j}$ of the identified modes $\lambda_{j}$, and the nonlinearity index Index 1 . The linear modes (Columns 1 and 2) will have many state variables involved. The largest linear participation factors for each mode are reported in column 3 and indicate state variables which contribute most to the mode. The second order mode contribution is a combination of the magnitudes of the second order terms (column 5) and their nonlinear participation factors (column 7). The determination of the groupings of the machines in the system requires consideration of both the linear and second order terms, as they contribute to the response. The two indices described above (columns 3 and 6) are useful in this process. An Index 1 value of 1 would indicate no contribution from the second order terms. The grouping procedure first consist of examining the linear term mode. We then use the highest nonlinear second order term ((b) in column 5) to determine the extent of the nonlinear interaction and the modes which contribute the most. Index 2 is then used to determine if the contribution from the second order terms is large. Finally the participation factors both linear and nonlinear are used to determine the machines in the interacting modes.

The data is presented in Table 2. From this table, we can make the following observations. (gen. * means generator at bus *).

1. Generator. 3597 (Column 4) has the highest participation in Mode 15. Mode pairs (59, 59), and $(15,101)$ have the highest nonlinear interaction (Column 5). These mode pairs will have the highest participation from Gen. 3596 (Mode 59, Column 4)), and Gen. 3597( Mode 15. Column 4) since the second order participation factors (Column 7 (c)) are small compared to the linear terms. We also note that these two interactions have a high value of Index2, indicating a large value of the second order coefficient in comparison to the linear term coefficient. As a result of this analysis, we get coupling between generators 3597 and 3596. In 
Table 2. Results of Normal Forms Analysis

\begin{tabular}{|c|c|c|c|c|c|c|}
\hline Mode & $\omega_{j}(\mathrm{rad} / \mathrm{sec})$ & Index 1 & $\begin{array}{l}\text { Largest linear } \\
\text { participation factor } \\
\text { (a) } \\
\text { Gen. \# (a) (variable) }\end{array}$ & $\begin{array}{l}\text { Largest nonlinear (second } \\
\text { order )interaction with } \\
\text { inertial mode (b) } \\
k, \ell \quad \text { (b) }\end{array}$ & Index 2 & $\begin{array}{l}\text { Largest second order } \\
\text { participation factor (c) } \\
\text { Gen.\#(c) (variable) }\end{array}$ \\
\hline 15 & 22.147 & 6.325 & $3597(0.49)(\delta)$ & $\begin{array}{c}59,59(5.21) \\
15,101(4.18)\end{array}$ & $\begin{array}{l}4.28 \\
3.44\end{array}$ & $\begin{array}{l}3596(0.0094)(\delta) \\
3597(0.0416)(\delta)\end{array}$ \\
\hline 17 & 19.847 & 3.804 & $3585(0.43)(\delta)$ & $\begin{array}{ll}17,101 & (1.37) \\
17,105 & (0.81)\end{array}$ & $\begin{array}{l}0.569 \\
0.336\end{array}$ & $\begin{array}{l}3585(0.0038)(\delta) \\
3581(0.4511)\left(E_{d}\right)\end{array}$ \\
\hline 19 & 17.112 & 2.411 & $\begin{array}{l}3586(0.37)(\delta) \\
3581(0.19)(\omega)\end{array}$ & $59,105(1.25)$ & 1.069 & $3581(0.7250)\left(E_{d}\right)$ \\
\hline 21 & 15.319 & 3.511 & $\begin{array}{l}3600(0.35)(\delta) \\
3587(0.15)(\delta)\end{array}$ & $23,105(1.32)$ & 0.559 & $3581(0.5748)\left(E_{d}\right)$ \\
\hline 23 & 15.122 & 2.922 & $\begin{array}{l}3587(0.32)(\omega) \\
3600(0.13)(\delta)\end{array}$ & $\begin{array}{ll}59,60 & (0.76) \\
59,59 & (0.70)\end{array}$ & $\begin{array}{l}0.135 \\
0.124\end{array}$ & $\begin{array}{l}3596(0.0661)(\delta) \\
3596(0.0094)(\delta)\end{array}$ \\
\hline 27 & 5.080 & 2.683 & $3590(0.25)(\delta)$ & $\begin{array}{ll}30,59 & (1.53) \\
50,60 & (1.11)\end{array}$ & $\begin{array}{l}0.307 \\
0.222\end{array}$ & $\begin{array}{l}3590(0.0496)(\delta) \\
3596(0.0661)(\delta)\end{array}$ \\
\hline 29 & 5.755 & 11.340 & $3590(0.20)(\omega)$ & $\begin{array}{cc}30,59 & (7.20) \\
29,101 & (4.07)\end{array}$ & $\begin{array}{l}0.98 \\
0.56\end{array}$ & $\begin{array}{l}3590(0.0496)(\delta) \\
3590(0.0121)(\delta)\end{array}$ \\
\hline 33 & 13.867 & 5.837 & $3595(0.49)(\delta)$ & $\begin{array}{c}33,101 \quad(3.14) \\
59,60(0.64)\end{array}$ & $\begin{array}{l}1.53 \\
0.313\end{array}$ & $\begin{array}{l}3595(0.0332)(\delta) \\
3596(0.0661)(\delta)\end{array}$ \\
\hline 35 & 7.567 & 4.148 & $3598(0.32)(\delta)$ & $35,101 \quad(1.76)$ & 0.963 & $3598(0.0171)(\delta)$ \\
\hline 45 & 9.062 & 6.506 & $3591(0.28)(\delta)$ & $\begin{array}{cc}45,101 & (2.64) \\
27,29 & (1.48)\end{array}$ & $\begin{array}{l}1.16 \\
0.655\end{array}$ & $\begin{array}{l}3591(0.0096)(\delta) \\
3590(0.0054)(\delta)\end{array}$ \\
\hline 57 & 11.293 & 11.700 & $3592(0.29)(\omega)$ & $\begin{array}{l}29,29(10.46) \\
57,101(6.98)\end{array}$ & $\begin{array}{l}3.56 \\
2.35\end{array}$ & $\begin{array}{l}3581(0.0413)(\delta) \\
3592(0.0194)(\delta)\end{array}$ \\
\hline 59 & 11.508 & 83.324 & $3596(0.42)(\omega)$ & $\begin{array}{cc}29,29 & (66.16) \\
59,101 & (42.83)\end{array}$ & $\begin{array}{l}2.49 \\
1.61\end{array}$ & $\begin{array}{l}3581(0.0413)(\delta) \\
3581(0.0105)(\delta)\end{array}$ \\
\hline 63 & 13.272 & 1.151 & $3599(0.426)(\omega)$ & $\begin{array}{c}63,101 \quad(1.36) \\
59,60(1.13)\end{array}$ & $\begin{array}{l}0.304 \\
0.253\end{array}$ & $\begin{array}{l}3599(0.0022)(\delta) \\
3596(0.0661)(\delta)\end{array}$ \\
\hline 83 & 12.410 & 7.063 & $3588(0.41)(\omega)$ & $\begin{array}{ll}29,29 & (4.89) \\
27,29 & (3.86)\end{array}$ & $\begin{array}{l}1.22 \\
0.969\end{array}$ & $\begin{array}{l}3581(0.0413)(\delta) \\
3590(0.0054)(\delta)\end{array}$ \\
\hline
\end{tabular}

the following observations we apply the same reasoning process.

2. Generator. 3585 has the highest participation in Mode 17. Mode pairs $(17,101)$ and $(17,105)$ have high nonlinear interaction. Gen. 3585 participates in mode pair $(17,101)$. However, the mode pair $(17,105)$ has significant participation from Gen. 3581. The values of Index 2 for these interactions are moderate indicating some coupling between generators 3585 and 3581 .

3. Generators. 3586 and 3581 have the highest participation in Mode 19. The mode pair (59, 105) will have the highest participation from Gen. 3581 followed by Gen. 3596 since, the second order participation factor for this mode pair is significantly larger than the highest linear participation factor. We also note that this mode pair has a high value of Index 2 . As a result, we see a strong coupling between generators 3586 , 3581 , and 3596.

4. Generators. 3587 and 3600 have the highest participation in both Modes 21 and 23 . For Mode 21, the mode pair $(23,105)$ has the highest second order term. The value of Index 2 for this interaction is moderate and shows coupling between these two generators to be strong. In addition the second order participation factor indicates a strong presence of Gen. 3581. For Mode 23, we note weak Index 2 is small for mode pairs $(59,60)$ and $(59,59)$ indicating weak coupling between these generators and the Gen. 3596 which participates in Mode 59. As a result, we obtain coupling between generators 3587 , 3600 , and 3581.

5. Generator 3595 has the highest participation in Mode 33, the mode pair $(33,101)$ has the highest second order term. This does not indicate any 
new coupling. The mode pair $(59,60)$ has a moderate value of Index2, indicating some coupling between Gen. 3595 and Gen 3596 which has the highest in Mode 59. As a result we obtain coupling between generators 3595 and 3596.

6. Generator 3596 has the highest participation in Mode 59. The mode pairs $(29,29)$ and $(59,101)$ have strong nonlinear interaction with this mode. The mode pair $(29,29)$ indicates a coupling between Gen. 3596 and Gen. 3590 which has the highest participation in Mode 29, however we observe that Mode 15 has a stronger coupling to Mode 59 as indicated by the higher value of Index 2 . Hence, we consider only the mode pair $(59,101)$ which does not give any further information regarding coupling.

7. Generator 3598 has the highest participation in Mode 35. This mode does not have any strong interactions. As result it forms a separate group. This generator is in close physical proximity to Gen. 3599. However, the nonlinear behavior indicates that it does not interact with any other machine. The results of the nonlinear analysis are confirmed by the time simulation results.

8. Generator 3599 has the highest participation in Mode 63. The mode pairs $(63,101)$ and $(59,60)$ have the highest second order term. These couplings are moderate as indicated by the values of Index2. Gen. 3596 has the highest participation in Mode 59 indicating some coupling between generator 3599 and 3596 .

From the machine couplings identified in observations 1-8, we form the first group of generators as:

Group 1: generators $3581,3585,3586,3587,3595$, $3596,3597,3599,3600$

Group 2: generator 3598

Using similar arguments in modes 45,57 , and 83 of Table 2, we form the third group of generators as:

Group 3: generators 3588, 3590, 3591, 3592

Figure 1, depicts the grouping of the generators in the Manitoba Hydro system with boxes drawn around the groups.

Time simulations conducted on the full Manitoba Hydro system do indicate the presence of the modes identified in the analysis. Figure 2, shows the relative angle plots of some key generators in the Manitoba Hydro system following the loss of the dc system and tripping of various lines. We see from the plot that Gen. 3587(Selkirk) and 3581(Dorsey SC 7-9) do form a group. In addition, Gen. 3590(Kelsey) and 3591(Grand Rapids(static)) also form a group. The simulation also shows the Gen. 3598 (Seven Sisters) does not belong to the two groups. This has been clearly shown by the normal forms analysis conducted above. This time domain result confirms the grouping prediction made by the method of normal forms. In the period between the loss of the dc system, and the tripping of lines the predicted groupings accurately describe the system dynamic behavior.

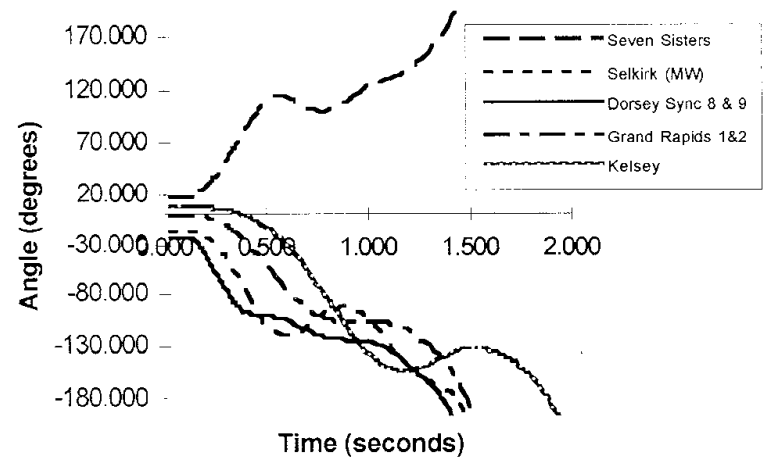

Figure 2 Relative Rotor Angle Plots

\section{Conclusions}

Based on the analysis presented above, and other cases analyzed but not presented in this paper, the following conclusions can be drawn:

- The analysis indicates that there is considerable nonlinear interaction between the fundamental modes of oscillation in the Manitoba Hydro system, and the normal forms analysis using second order terms captures this interaction.

- The results of the normal forms analysis on several cases of the Manitoba Hydro system provide the following insight into system behavior:

A. The normal forms analysis captures all the important fundamental modes of oscillation, and associates all the generators which are represented in the Manitoba Hydro system with appropriate modes.

B. The nonlinear interaction index accurately determines the fundamental modes of oscillation, which have large nonlinear interaction.

C. The second order coefficients associated with the modes identified by the nonlinear index 
correctly identify the modes interacting with the fundanental mode in the linear term.

D. The second order participation factors and the linear participation factors correctly identify the state variables of the system associated with the interacting modes.

- The generator grouping obtained is established using the nonlinear interactions and the analysis from the method of normal forms. This grouping does not imply any coherency. It is a measure of the inherent dynamic behavior of the system including nonlinear effects. The second order nonlinear effects describe the behavior of fundamental modes that are "near" second order resonant, i.e. the sum of two modes is close to a third mode. We do not see this grouping from linear analysis which is essentially provided in Columns 1, 2, and 4 of Table 2 . We observe that the participation factors associated with the various modes do not group the machines in the groups obtained by the nonlinear analysis.

- The islanding scheme could be envisioned as follows. The three groups formed in the Manitoba Hydro system are shown in Figure 2. This indicates that if the tie lines connecting these three groups are opened, then the desired islands could be formed. In addition the Saskatchewan machines form separate groups. Hence the tie lines with Saskatchewan would also need to bis opened.

\section{Acknowledgment}

This work was supported by EPRI(Project WO900037) and Manitoba Hydro, to whom grateful acknowledgment is made.

\section{References}

[1] Lin, C-M, V. Vittal, W. Kliemann, and A. A. Fouad, "Investigation of Modal Interactions and its Effects on Control Performance in Stressed Power Systems Using Normal Form of Vector Fields," IEEE. Trans. on Power Systems 11, No. 2, pp. 781-787. May 1996.

[2] Thapar, J., V. Vittal, W. Kliemann, and A. A. Fouad, "Application of the Normal Form of Vector Fields to Predict Interarea Separation in Power Systems," Paper no. 96SM517-3, IEEE Summer Power Meeting, Denver, Colorado, July 1996.

[3] Starrett, S. K., and A. A. Fouad, "Nonlinear Measures of Mode Machine Participation," Paper no. 97SM697, IEEE Summer Power Meeting, Berlin, Germany, July 1997.
[4] Fouad, A. A., V. Vittal, W. Kliemann, et al. "Analysis of Stressed Interconnected Power Networks." TR-103704, Research Project 8010-28, EPRI Final Report, March 1994.

[5] Fouad, A. A., V. Vittal, W. Kliemann, et al. "Nonlinear Power System Behavior Using Normal Forms: Extension of Linear System Analysis via Higher Order Correction." TR-107798, Research Project 8050-05, EPRI Final Report February 1997.

[6] Anderson, P. M., and A. A. Fouad, Power System Control and Stability, IEEE Press, New York, 1993.

[7] Perez-Arriaga, I. J.., G. C. Verghese, and F. C. Schweppe, "Selective Modal Analysis with Applications to Electric Power Systems. Part 1; Heuristic Introduction," IEEE Trans. on PAS, 101, pp. 3117-3125, September 1982.

[8] EPRI Report, "Extended Transient-midterm Stability Program (ETMSP)," Version 3.1, Final Report, Vol. 1, RP1208, 1994.

\section{Biographical Sketches}

V. Vittal (S'78- F'97) is a Professor in the Electrical and Computer Engineering Department at Iowa State University. He is a recipient of the 1985 Presidential Young Investigator Award.

W. Kliemann is a Professor in the Mathematics Department at Iowa State University. He is a member of SIAM, AMS, IMS, and DMV.

Y.X Ni (SM'94) is an Associate Professor in the Electrical \& Electronic Engineering Department at the University of Hongkong. She received her B.S., M.S., and $\mathrm{Ph} . \mathrm{D}$. degrees all in Electrical Engineering from Tsinghua University, Beijing China

D.G.Chapman (SM'90) is the Manager, System Performance at Manitoba Hydro. He received his B.Sc. (Electrical Engineering) from the University of Manitoba in 1968 and the Ph.D. in Automatic Control from the University of London (Imperial College) in 1973. He is a Professional Engineer in the Province of Manitoba.

A.D.Silk is a system studies engineer in System Performance at Manitoba Hydro. He received his B.Sc. (Computer Engineering) from the University of Manitoba in 1985. He is a Professional Engineer in the Province of Manitoba.

D. J. Sobajic (SM) is a Manager of Power Electronic Development in the Power Delivery Group at EPRI. He received his Dipl. Ing. EE and MSEE from the University of Belgrade and the Ph.D. from Case Western Reserve University. He is a member of INNS. 